Chronic Obstructive Pulmonary Diseases: Journal of the COPD Foundation

\author{
Letter to the Editor
}

\title{
Smoking Cessation Amid the Coronavirus 2019 Pandemic: Making Every Contact Count
}

\author{
Syed Shahzad Hasan, $\mathrm{PhD}^{1}$ Chia Siang Kow, MPharm ${ }^{2}$ Keivan Ahmadi, $\mathrm{PhD}^{3}$
}

\begin{abstract}
Abbreviations: coronavirus disease 2019, COVID-19; severe acute respiratory syndrome coronavirus 2, SARS-CoV-2; angiotensinconverting enzyme 2, ACE2

Funding Support: not applicable

Citation: Hasan SS, Kow CS, Ahmadi K. Smoking cessation amid the coronavirus 2019 pandemic: making every contact count. Chronic Obstr Pulm Dis. 2020;7(4):300-302. doi: https://doi.org/10.15326/jcopdf.7.4.2020.0171
\end{abstract}

1 Department of Pharmacy, University of Huddersfield,

Huddersfield, United Kingdom and School of Biomedical

Sciences and Pharmacy, University of Newcastle, Callaghan,

Australia

2 School of Postgraduate Studies, International Medical University, Kuala Lumpur, Malaysia

3 Lincoln Medical School, Universities of Nottingham and Lincoln, Lincolnshire, United Kingdom

\section{Address correspondence to:}

Chia Siang Kow, MPharm

School of Postgraduate Studies

International Medical University

Kuala Lumpur, Malaysia

Email: chiasiang_93@hotmail.com

\section{Keywords:}

Coronavirus disease 2019, COVID-19, smoking cessation

\section{Dear Editor}

Recently, YouGov and the campaign group, Action on Smoking and Health, published the results of their survey on the impact of coronavirus disease 2019 (COVID-19) on the smoking behavior of current adult smokers. ${ }^{1}$ The study, which interviewed over 1000 adult participants residing in the United Kingdom during April 2020, revealed some positive findings with regards to smoking behavior in association with the COVID-19 pandemic. Specifically, 36\% of smokers had reduced the amount of tobacco or cigarettes smoked while $8 \%$ of smokers had tried to quit smoking. Collectively, the proportion of smokers who had reduced or tried to quit smoking was more than the proportion of smokers (20\%) who had purchased tobacco or cigarettes in larger quantities -bulk-buying or stockpiling-because of COVID-19 associated lockdowns. Though lesser in proportion, it is also encouraging to observe that $2 \%$ of smokers had quit smoking in the last 4 months, solely or partly due to COVID-19.

These positive findings may be due to media coverage in which smokers have been predicted to have a higher risk of COVID-19 acquisition than nonsmokers. Though no studies as yet have proven such an association, it has been suggested that smokers could be more vulnerable to acquiring COVID-19, as their fingers (and possibly contaminated tobacco or cigarettes) are often in contact with their lips, which increases the likelihood of the transmission of severe acute respiratory syndrome coronavirus 2, (SARSCoV-2), the causative pathogen of COVID-19, from hand to mouth. ${ }^{2}$

Indeed, similar to individuals with chronic obstructive pulmonary disease, smokers have been reported to have an increased expression of angiotensin-converting enzyme 2 (ACE2) in the bronchial epithelial cells of the lower respiratory tract, which act as the port of entry for SARS-CoV-2 into host cells. ${ }^{3}$ Such a finding not only indicates the possibility for a heightened risk of COVID-19 acquisition among smokers but also an increased risk of developing more severe illness, should they acquire COVID-19, due to a possibly higher viral load along with a more pronounced cytokine storm syndrome arising from the immune-mediated clearance of this overwhelming viral load. Thus far, findings on the association between smoking and severity of 
COVID-19 are mixed, though a large-scale study on 1099 patients with COVID-19 from 552 hospitals in China reported a higher proportion of current and former smokers who required intensive care unit admission and/or mechanical ventilation, or had died, than non-smokers. ${ }^{4}$ Moreover, smokers constituted a higher proportion compared to non-smokers among the patients who had severe manifestations of COVID-19.

All clinicians around the world should seize this golden opportunity to encourage their active smoking patients or clients to quit smoking, citing the possible increased risk of contracting COVID-19 and its associated severe illness among smokers. Many smokers may already have the intention to quit during the COVID-19 pandemic, as reported in the aforementioned survey, either because of a fear of contracting COVID-19 or the difficulty in purchasing cigarettes amid COVID-19 associated lockdowns. Every clinician, especially those working in the community or primary care, is in a unique position during the current pandemic to fully utilize the established 5-step treatment framework for smoking cessation-the " 5 As approach": ask about tobacco use, advise quitting, assess readiness to quit, assist smokers ready to quit, and arrange follow-up. ${ }^{5}$ For patients or clients who are prepared to stop smoking or at least reduce the number of cigarettes smoked per day, pharmacotherapies for smoking cessation, including nicotine replacement therapy, varenicline, and bupropion can be offered. ${ }^{6}$

While it can be challenging to provide a smoking cessation service in a busy practice, which may be made worse amid the COVID-19 pandemic given the need to observe social distancing, there is clear evidence that brief ( $<5$ minutes) advice to quit smoking at each patient or client encounter can increase smoking abstinence rates, regardless of whether patients are prepared for immediate quitting.
A 2013 Cochrane systematic review ${ }^{7}$ of 42 trials with over 31,000 smokers concluded that a brief advice intervention could increase the baseline unassisted quit rate of $2 \%-3 \%$ by a further $1 \%-3 \%$. Clinicians who have concerns about patient follow-up difficulties created by the COVID-19 pandemic, such as imposed social distancing rules and lockdowns, could avail themselves of telehealth approaches. A follow-up as simple as a telephone call may be useful to increase patients' smoking abstinence rates. A 2019 Cochrane systematic review $^{8}$ of 104 trials reported evidence of moderate certainty that proactive telephone counseling increases quit rates in smokers. Clinicians could also refer their patients for external resources including telephonic quitline support if follow-up proves not feasible. This would also allow for the involvement of other trained health care professionals to jointly provide smoking cessation services. The same systematic review reported moderate certainty evidence that proactive telephone counseling aids smokers who seek help from quitlines. ${ }^{8}$

In conclusion, we would like to once again stress that clinicians should engage smokers in the process of smoking abstinence at every opportunity amid the COVID-19 pandemic, since the readiness for smoking cessation may have been facilitated by the fear of contracting COVID-19. This includes patients who have not been diagnosed by COVID-19 as yet and patients who have had COVID-19 and have since recovered from acute illness. In addition, controversial smoking cessation strategies such as electronic cigarettes should be discouraged as there is documented evidence regarding the vapingrelated pulmonary diseases, which may further reduce pulmonary reserve in smokers. ${ }^{9}$

\section{Declaration of Interest}

The authors have nothing to declare. 


\section{References}

1. YouGov, Action on Smoking and Health. COVID-19 and smoking. YouGov.co.ukwebsite. Published May 5, 2020. Accessed May 31, 2020. https://docs.cdn.yougov.com/h3fyf97ah6/YG-Archive-05052020ASHcovid19.pdf

2. World Health Organization (WHO). Q\&A: Tobacco and COVID-19. WHO website. Published May 27, 2020. Accessed May 31, 2020. https://www.who.int/news-room/q-a-detail/q-a-on-tobacco-and-covid-19

3. Leung JM, Yang CX, Tam A, et al. ACE-2 expression in the small airway epithelia of smokers and COPD patients: implications for COVID-19. Eur Respir J. 2020;55(5):2000688.

doi: https://doi.org/10.1183/13993003.00688-2020

4. Guan WJ, Ni ZY, Hu Y, et al. Clinical characteristics of coronavirus disease 2019 in China. N Engl J Med. 2020;382(18):1708-1720.

5. Agency for Healthcare Research and Quality (AHRQ). Five Major Steps to Intervention (The "5 A's"). AHRQ website. Published December 2012. Accessed May 31, 2020. https://www.ahrq.gov/prevention/ guidelines/tobacco/5steps.html

6. Barua RS, Rigotti NA, Benowitz NL, et al. 2018 ACC Expert consensus decision pathway on tobacco cessation treatment: a report of the American College of Cardiology Task Force on Clinical Expert Consensus Documents. J Am Coll Cardiol. 2018;72(25):3332-3365. doi: https://doi.org/10.1016/j.jacc.2018.10.027

7. Stead LF, Buitrago D, Preciado N, Sanchez G, Hartmann-Boyce J, Lancaster T. Physician advice for smoking cessation. Cochrane Database Syst Rev. 2013;2013(5): CD000165. doi: https://doi.org/10.1002/14651858.CD000165.pub4

8. Matkin W, Ordóñez-Mena JM, Hartmann-Boyce J. Telephone counselling for smoking cessation. Cochrane Database Syst Rev. 2019;5(5):CD002850.

doi: https://doi.org/10.1002/14651858.CD002850.pub4

9. Stanbrook MB, Drazen JM. Vaping-induced lung disease - a look forward by looking back. N Engl J Med. 2020;382(17):1649-1650.

doi: https://doi.org/10.1056/NEJMe2004876 